\title{
Influence of Tonifying Kidney and Spleen, Detoxification of Throat Method to MMP-9,TIMP-1 of IgA Nephropathy Model Rat Di ZOU ${ }^{1, a}$, Yin-pin WANG ${ }^{2}$, Tian-ying $\mathrm{CHANG}^{3}$ and Shou-lin ZHANG ${ }^{4 b^{*}}$
}

${ }^{1,2,3,4}$ Department of Nephrology, the First Affiliated Hospital to Changchun University of Chinese Medicine, Changchun, Jilin province, China,130021.

aaggiezou@163.com , bshoulin-z@163.com

* Shou-lin ZHANG

Keywords: Tonifying kidney and spleen, Detoxification of throat method, $\lg A$ nephropathy, Rat, MMP-9, TIMP-1.

\begin{abstract}
Purpose:To study the method of tonifying kidney and spleen, detoxification of throat in treatment of IgA nephropathy. Methods:72 Wistar rats were randomly divided into blank group, model group, Lotensin group, tonifying the kidney and spleen, detoxification of method of high, medium and low dose group (hereinafter referred to as TCM high, medium and low dose group). The IgAN rat model was established by oral administration of BSA and LPS intravenous injection method. Observed after treatment for 8 weeks rats in each phase of urine red blood cell count, 24h urinary protein (UTP) and renal tissue MMP-9, TIMP-1 changes. Results:1) compared with the model group, rats $24 \mathrm{~h}$ UTP has declined. The TCM medium dose group decreased significantly $(P<0.05)$; 2) compared with the model group, TCM in the treatment group the urine red blood cell count decreased significantly $(P<0.05)$. TCM medium dose group decreased significantly $(P<0.01)$; urinary red blood cell of lotensin group decreased $(P>0.05)$. 3) compared with the blank group, the content of MMP-9 in renal tissue of the rats in the model group decreased significantly, the content of TIMP-1 increased significantly, and MMP-9/TIMP-1 decreased $(P<0.05)$; 4) after taking drug, compared with the model group, treatment group content of MMP-9 significantly increased and TIMP-1 levels were significantly lower and MMP-9/TIMP-1 significantly increased $(P<0.05)$. TCM medium dose group was most significantly $(P<0.01)$. Conclusion:Tonifying kidney and spleen, detoxification of throat method can reduce the IgAN model rats with hematuria, proteinuria and increase MMP-9 and reduce the TIMP-1 in renal tissue, and MMP-9/TIMP-1 tends to be balance, the curative effect is better than that of Lotensin group.
\end{abstract}

\section{Introduction}

IgA nephropathy (IgAN) is a relatively common glomerular disease. The incidence rate is accounting for 30\% 40\% of primary glomerular disease in China[1]. After 20years end stage renal disease incidence rate is 15\% 40\%[2]. The main features of IgAN were hematuria and proteinuria.It is the first cause in primary glomerular disease which needs to accept the renal replacement therapy[1]. Tonifying kidney and spleen, detoxification of throat method is suggested by Chinese medicine master Jixue Ren, it is widely applied in clinical for more than 20 years, which can effectively reduce the IgAN patients with hematuria and proteinuria and delay the deterioration of renal function. In order to explore the mechanism of this method for treatment of IgAN, the author carried out the experiment research, the results reported as follows. 


\section{Experimental Materials}

\section{Experimental Animals}

Selected 6 weeks old healthy clean grade male Wistar rats 72, body weight 160 $200 \mathrm{~g}$, provided by the animal laboratory of Jilin University Bethune School of medicine.Experimental animal number: SCXK (Liao) 2013-000.

\section{Experimental Instruments, Reagents and Drugs}

Fluorescence microscopy TXM-500C,Shanghai Tian Sheng Instrument Co.,Ltd.; MMP-9 and TIMP-1 ELISA detection kit provided by the Shanghai Xinyu Biological Technology Co.,Ltd; Bovine serum albumin (BSA) and lipopolysaccharide (LPS), sigma company, USA; fluorescein labeled Rabbit anti rat IgA antibody, Beijing boisynthesis Biotechnology Co., Ltd. Lotensin provided by Beijing Novartis Pharmaceutical Co., Ltd. (country medicine number 1050423H0); tonifying kidney and spleen, detoxification of throat method (by radix rehmanniae, Radix Astragali, Radix Codonopsis, golden buckwheat, oroxylum indicum, puffball, Cercis chinensis, radix curcumae, Rosa laevigata, tuckahoe, Rhizoma imperatae, pollen Typhae etc.) fried by the Affiliated Hospital of Changchun University of traditional Chinese medicine preparation room.

\section{Experimental Methods}

\section{Method of Making Model}

Rats were randomly divided into blank group, model group, Lotensin group, TCM high, medium and low dose group.According to the method reported by Huiyu Lu 's[3] animal model,after ninth weeks each group selected 2 rats, the success of the model was verified by the test of the renal tissue.

\section{Grouping and Drug Validation}

After the model validation, control group were given Lotensin $10 \mathrm{mg} / \mathrm{kg}$ orally; high dose of TCM group was given $32 \mathrm{~g} / \mathrm{kg}$ irrigation stomach every day; medium dose of TCM group was given $16 \mathrm{~g} / \mathrm{kg}$ irrigation stomach; low dose of TCM was given $8 \mathrm{~g} / \mathrm{kg}$; blank group and model group only received Physiological saline $4 \mathrm{ml}$ every day. Drug validation continue for 8 weeks.

\section{Observation Index}

Observing the general state of rats, including body weight, hair color, urination and urine volume. Respectively in $0,4,6,8,10,12,14$ and 16 weeks, in a metabolic cage collecting $24 \mathrm{~h}$ urine, observing and recording the color of urine, urine volume, hematuria in frequency and severity, Urine samples being taken from 24 UTP and urinary sediment examination of urinary red blood cell count, using ELISA method to detect the renal tissue MMP-9 and TIMP-1 levels.

\section{Statistical Methods}

Using SPSS 19 statistical software, measurement data was descripted with $\left(\bar{x}_{ \pm \mathrm{s}}\right), P<$ 0.05 differences have statistical significance. 


\section{Experimental Results}

\section{The General Situation}

There were 7 rats died in the process of making model,and 2 rats of the each group selected for the validation of the model being successful. Blank group of rats in the experimental process of the general state has always been good. Other group 4 weeks later the rats appeared eating and activity decreased, dull hair color, weight loss, diarrhea and urine discoloration deep; in the 10th week drug treating rats general condition improved markedly, and body weight increased.

\section{Comparison of Urine Red Blood Cell Count in Rats of Each Group}

Table 1 showed compared with the blank group, the red blood cell count began to increase in the model group since sixth weeks $(P<0.05)$ and gradually increased with time $(P<0.01)$; compared with the model group, urinary red blood cell count of Lotensin group, was not significantly reduced $(P>0.05)$, but the TCM treatment group were since the beginning of the 12 week decreased gradually $(P<0.05)$.In the 16 week decreasing was more significantly $(P<0.05)$;compare between TCM groups,TCM medium dose group reduced significantly $(P<0.01)$.

Table 1 Comparison of urine red blood cell count of each group of rats before and after treatment (HP)

\begin{tabular}{|c|c|c|c|c|c|c|}
\hline Week & Blank group & Model group & lotensin group & High dose group & Medium dose group & Low dose group \\
\hline 0 & $4.82 \pm 0.52$ & $4.52 \pm 0.40$ & $3.24 \pm 0.33$ & $3.88 \pm 0.35$ & $4.09 \pm 0.63$ & $4.09 \pm 0.70$ \\
\hline 4 & $4.96 \pm 0.39$ & $5.30 \pm 0.93$ & $6.24 \pm 0.22$ & $5.23 \pm 0.63$ & $5.66 \pm 0.56$ & $5.49 \pm 0.42$ \\
\hline 6 & $4.77 \pm 0.53$ & $62.13 \pm 10.53^{\#}$ & $68.22 \pm 0.49^{\#}$ & $64.03 \pm 0.39^{\#}$ & $59.45 \pm 0.46^{\#}$ & $60.66 \pm 0.36^{\#}$ \\
\hline 8 & $4.42 \pm 0.34$ & $138.20 \pm 15.25^{\# \#}$ & $129.60 \pm 0.40^{\# \#}$ & $132.15 \pm 0.46^{\# \# ~}$ & $140.72 \pm 0.48^{\# \#}$ & $134.86 \pm 0.50^{\# \# ~}$ \\
\hline 10 & $4.21 \pm 1.21$ & $133.21 \pm 1.24^{\# \#}$ & $132.79 \pm 1.72$ & $126.22 \pm 1.50$ & $129.08 \pm 1.56$ & $125.20 \pm 1.22$ \\
\hline 12 & $4.11 \pm 1.14$ & $161.43 \pm 1.83^{\# \#}$ & $1558 \pm 1.61$ & $118.17 \pm 1.28^{\mathbf{\Delta}}$ & $108.56 \pm 1.45^{\mathbf{\Delta}}$ & $120.97 \pm 1.60^{\Delta}$ \\
\hline 14 & $4.29 \pm 1.26$ & $172.81 \pm 0.60^{\# \#}$ & $169.43 \pm 1.03$ & $99.73 \pm 0.58^{\star} \triangle$ & $87.63 \pm 1.01^{\wedge} \triangle$ & $95.85 \pm 1.15^{\mathbf{\triangle}}$ \\
\hline 16 & $4.93 \pm 1.27$ & $166.49 \pm 1.80^{\# \#}$ & $163.56 \pm 1.78$ & $88.33 \pm 1.20^{\Delta} \triangle$ & $79.56 \pm 0.58^{\wedge}$ & $84.90 \pm 1.40^{\mathbf{\Delta}}$ \\
\hline
\end{tabular}

Notes:compare with blank group, ${ }^{\#} P<0.05,{ }^{\# \#} P<0.01$; compare with model group, ${ }^{\mathbf{\Lambda}} P<0.05$; compare with lotensin group, ${ }^{\Delta} P<0.05$; compare between TCM groups, ${ }^{\bullet} P<0.05$.

\section{Comparison of 24h UTP in Rats of Each Group}

Table 2 shows compared with the blank group,the model group from six weeks $24 \mathrm{~h}$ UTP began increased $(P<0.05)$ and increased with the time $(P<0.01)$; compared with the model group, treatment group from the 10 weeks $24 \mathrm{~h}$ UTP began to decline.the 16th week decline significantly $(P<0.05)$;compare between TCM groups, the medium group was more significant, and the therapeutic effect was better than the lotensin group. $(P<0.01)$.

Table2 Comparison of $24 \mathrm{~h}$ urinary protein in rats before and after treatment $(\mathrm{mg} / 24 \mathrm{~h})$

\begin{tabular}{|c|c|c|c|c|c|c|}
\hline Week & Blank group & Model group & lotensin group & High dose group & Medium dose group & Low dose group \\
\hline 0 & $25.65 \pm 0.56$ & $32.21 \pm 0.45$ & $32.11 \pm 0.44$ & $32.52 \pm 0.43$ & $43.26 \pm 0.73$ & $46.55 \pm 0.36$ \\
\hline 4 & $43.23 \pm 0.39$ & $77.15 \pm 0.71$ & $82.83 \pm 0.46$ & $69.85 \pm 0.34$ & $76.56 \pm 0.23$ & $80.26 \pm 0.22$ \\
\hline 6 & $49.87 \pm 0.52$ & $122.15 \pm 0.99^{\# \# ~}$ & $130.55 \pm 0.22^{\#}$ & $136.56 \pm 0.68^{\#}$ & $135.80 \pm 0.43 \#$ & $14.69 \pm 0.66 \#$ \\
\hline 8 & $48.64 \pm 0.31$ & $626.30 \pm 15.24^{\# \# ~}$ & $632.30 \pm 048^{\# \#}$ & $660.50 \pm 0.22^{\# \#}$ & $659.51 \pm 0.57$ \#\# & $645.30 \pm 0.36 \# \#$ \\
\hline 10 & $40.70 \pm 1.24$ & $772.13 \pm 1.16^{\# \#}$ & $626.85 \pm 1.10^{\wedge}$ & $610.82 \pm 1.32^{\wedge}$ & $542.71 \pm 1.19^{\wedge}$ & $564.54 \pm 1.49^{\wedge}$ \\
\hline 12 & $46.19 \pm 1.17$ & $978.69 \pm 1.63^{\# \#}$ & $589.30 \pm 1.20^{\wedge}$ & $527.48 \pm 1.71^{\wedge}$ & $410.71 \pm 1.86^{\wedge}$ & $512.25 \pm 1.73^{\wedge}$ \\
\hline 14 & $46.34 \pm 1.65$ & $938.23 \pm 1.41^{\# \#}$ & $516.61 \pm 1.50^{\wedge}$ & $454.63 \pm 1.62^{\wedge}$ & $406.19 \pm 2.02^{\wedge}$ & $461.53 \pm 1.58^{\Delta}$ \\
\hline 16 & $48.01 \pm 0.68$ & $987.85 \pm 1.15^{\# \#}$ & $431.21 \pm 0.62^{\wedge}$ & $370.10 \pm 0.52^{\wedge} \triangle$ & $317.90 \pm 0.98^{\wedge} \bullet$ & $399.16 \pm 0.63^{\Delta}$ \\
\hline
\end{tabular}

Notes:compare with blank group, ${ }^{\#} P<0.05,{ }^{\#} P<0.01$; compare with model group, ${ }^{\mathbf{\Lambda}} P<0.05$; compare with lotensin group, ${ }^{\triangle} P<0.05$; compare between TCM groups, ${ }^{\bullet} P<0.05$. 


\section{MMP-9 and TIMP-1 in kidney tissue of rats after treatment}

Table 3 shows compared with the blank group, the MMP-9 in the model group significantly decreased,TIMP-1 significantly increased, MMP-9/TIMP-1<1 $(P<0.05)$. Compared with model group, MMP-9 significantly increased in each group. TIMP-1 significantly decreased. TIMP-1/MMP-9 significantly increased $(P<0.05)$. The traditional Chinese medicine medium dose group was the most significant $(P<0.01)$.

\begin{tabular}{|c|c|c|c|}
\hline Group & MMP-9 ( pg / mL) & TIMP-1 ( pg / mL) & MMP-9 /TIMP-1 \\
\hline Blank group & $2.19 \pm 0.77$ & $1.99 \pm 0.16$ & $1.10 \pm 0.016$ \\
\hline Model group & $1.61 \pm 0.66^{\#}$ & $2.43 \pm 0.66^{\#}$ & $0.66 \pm 0.041^{\#}$ \\
\hline Lotensin group & $1.81 \pm 0.44^{\boldsymbol{\Lambda}}$ & $2.11 \pm 0.82^{\mathbf{\Lambda}}$ & $0.87 \pm 0.01^{\boldsymbol{\Lambda}}$ \\
\hline High dose group & $1.93 \pm 0.79^{\wedge}$ & $2.06 \pm 0.46^{\mathbf{\Lambda}}$ & $0.93 \pm 0.029^{\wedge}$ \\
\hline Medium dose group & $2.03 \pm 0.70^{\mathbf{\Lambda}}$ & $2.01 \pm 0.60^{\boldsymbol{\Lambda}}$ & $1.01 \pm 0.660^{\mathbf{\Lambda}}$ \\
\hline Low dose group & $1.95 \pm 0.88^{\wedge}$ & $2.03 \pm 0.29^{\wedge}$ & $0.96 \pm 0.840^{\wedge}$ \\
\hline
\end{tabular}

\section{Conclusion}

At present, it is considered that the main cause of $\operatorname{IgAN}$ is that the $\operatorname{IgA}$ molecules and the large molecular immune complexes are deposited on the surface of the glomerular, which lead to glomerular sclerosis and renal tubular interstitial fibrosis, which eventually lead to renal failure[4]. A large number of studies at home and abroad show that [5-7] glomerular sclerosis and renal interstitial fibrosis are important factors influencing the prognosis of renal disease. In this process, the increase of extracellular matrix $(\mathrm{ECM})$ is the characteristic change of chronic progressive glomerular disease, and it is also the main pathological basis of glomerular sclerosis[8]. Studies[9] have indicated that the most important class of proteases in the decomposition of ECM are matrix metalloproteinases (MMPs). MMP-9 belongs to gelatinase, it can degrade IV collagen of the basement membrane. MMPs and tissue inhibitor of metalloproteinase (TIMP) are the main factors in regulating ECM synthesis and degradation balance. Studies have shown that MMP-9 is involved in the progression of renal disease in IgA nephropathy, and is closely associated with the production of proteinuria [10-12].Animal experiments have confirmed that MMP-9 plays an important role in the pathogenesis of glomerular disease[13]. TIMP-1 may be one of the contributing factors to the formation of renal interstitial fibrosis, and it is involved in the whole process of the formation of fibrosis[14]. In regulation of MMP-9/TIMP-1,ECM is in a state of dynamic equilibrium, when the external factors leads to the imbalance of dynamic regulation of MMP-9/TIMP-1, ECM will accumulate and aggravate glomerular sclerosis and renal tubule fibrosis, resulting in loss of renal function. So the formation and degradation of ECM are the keys to the development of renal disease. And TIMP-1/MMP-9 plays an important role in regulating it.

Master of traditional Chinese medicine Professor Jixue Ren think that the pathogenesis core of this disease is the the deficiency of spleen and kidney, and toxin stagnation throat is causative factors of the disease[15-17]. The kidney is the origin of congenital constitution and the spleen is acquired, kidney and spleen determine the body's resistance strength. "Lingshu" pointed out that "the kidney meridian of foot Shaoyin meridian,...... The straight from the renal tubular liver and diaphragm, lung, throat cycle, relying on the tongue ".Throat is kidney lung collaterals for access to the evils of the portal and toxin from the nose and mouth entrenched in the throat forming 
milk moth from blood invasion in the kidney. Fuxie formation long and to poison living in the palace of kidney forming a vicious cycle of throat, lung, kidney. Therefore, Nephritis is closely related to the occurrence and relapse and illness aggravating and throat, so professor Ren raised tonifying the kidney and strengthening the spleen, detoxification of throat.

This study showed that the treatment group after treatment can significantly reduce the IgAN rats $24 \mathrm{~h}$ UTP content. Chinese medicine group can significantly reduce the urine red blood cell count, Lotensin group was inefficacy in the treatment of urinary red blood cell count; each treatment group make MMP-9 in renal tissue increased significantly, TIMP-1 was significantly decreased, MMP-9/TIMP-1 obviously increased and tends to the equilibrium. Presumably, tonifying kidney and spleen, detoxification of throat method is through the regulation of MMP-9/TIMP-1 balance to reduce glomerular sclerosis and renal tubule interstitial fibrosis and thus delay the deterioration of renal function.

\section{Acknowledgement}

This research was financially supported by project as follows: Jilin Province Science and Technology Department of Natural Science Fund Project (No. 2015101214JC). Jilin Province Administration of traditional Chinese medicine science and technology project (No. 2014-zd7).Jilin Province Education Department "Twelfth Five Year Plan" science and technology research project (No. 65 of 2014);

\section{Reference}

[1] Peng Yajun, Li Xuhua, He Zeyun, et al. The effect of six ingredient Di Huang Decoction on the expression of SMA and FN in rats with $\operatorname{IgA}$ nephropathy[J](in Chinese).Journal of Hunan University of Chinese Medicine. 2015,35( 11) : 22- 26.

[2] BARRATT J,FEEHALLY J.IgA nephropathy [J].J Am Soc Nephrol, 2005, 16:2088- 2097.

[3] Lu Huiyu, Zhang Qiaoling, Jiang Xiaoyun, et al. Establishment of the rat model of IgAN [J] (in Chinese). Journal of Chinese medical science, 2011, 11 (6): 1264- 1267

[4] WADA J, SNGIYAMA H, MAKINO H. Pathogenesis of IgA nephropathy [J] Semin Nephrol,2003,23(6):556- 563.

[5] Peng Youming, Liu Fuyou. Study of renal Department of internal medicine[M] (in Chinese).Changsha: Central South University press. 2006: 40.

[6] BOGENSCHUTZ O, BOHLE A, BATZ C, et al. IgA Nephritis: on the importance of morphological and clinical parameters in the long oterm prognosis of 239 patients[J].Am J Nephrol,1990, 10: 137.

[7] ALAMARTINE E, SABATIER J C, GUERIN C, et a1. Prog-nostic fa- vors in mesangial IgA glomerulonephritis: all extensive studywith univariate and multivariate analysis[J].Am J Kidney Dis, 1991,18:12.

[8] Wang Haiyan. Kidney disease. Beijing: People's Medical Publishing House, China.2008: 241-243. 
[9] Guo Xin rang, Wang Qingwen. Study on the relationship between matrix metalloproteinases and rheumatic diseases[J] (in Chinese). Shanxi Journal of medicine. 2011,40(8):790- 792.

[10] Chen Yan, Xiong Peihua, Zhang Ling, et al. The expression of matrix metalloproteinase 9 and tissue inhibitor of metalloproteinase 1 in serum of patients with IgA nephropathy and lupus nephritis [J] (in Chinese). Journal of Soochow University (Medical Sciences). 2009, 29(4) : 713- 714.

[11] Zhang Liping, Li Shaomei, Pei Huaying, et al.The significance of expression of matrix metalloproteinase-9 in podocyte injury in $\operatorname{IgA}$ nephropathy[J](in Chinese) . Chinese generalpractice. 2010,13(11) : 1182- 1184.

[12]THRAILKILL K M,CLAY BUNN R,FOWLKES J L.Matrix metalloproteinases: their potential role in the pathogenesis of diabetic nephropathy[J]Endocrine,2009,35(1) : 1- 10.

[13] SAKAMAKI Y,SASAMURA H,HAYASHI K,et al.Absence of gelatinase (MMP-9) or collagenase (MMP-13) attenuates adri-amycin induced albuminuria and glomerulosclerosis[J].Nephron Exp Nephrol,2010,115(2) : 22- 32.

[14] Zhao et al. Yang Dasheng. Guizhi Fuling Capsule on renal tubular interstitial fibrosis in rats with alpha smooth muscle actin (SMA), type IV collagen and transforming growth factor beta 1 expression effect $[\mathrm{J}]$ (in Chinese). Journal of Pediatrics. 2013,31(1) : 65- 66.

[15] Liu Yanhua, Ren Xijie, Wang Jian, et al. Professor Ren Jixue's application in the diagnosis and treatment of chronic renal wind related theory of the throat and kidney. Journal of traditional Chinese medicine[J] (in Chinese). 2015,56(4):283- 285.

[16] Tian Yudong, Ma Defeng, Ren Xijie.Professor Ren Jixue in the treatment of renal wind exogenous medicine experience[J] (in Chinese). China community doctors. 2014,30(28):93- 95.

[17] Yang Li. Professor Ren Jixue on glomerulonephritis in traditional Chinese medicine theory views[J] (in Chinese). Journal of Guangzhou University of Chinese Medicine. 2003,20(1):79- 81. 